Pacific Journal of Mathematic 


\title{
ON THE ZEROS OF CONVEX COMBINATIONS OF POLYNOMIALS
}

\author{
H. J. FELL
}

Given monic $n$th degree polynomials $P_{0}(z)$ and $P_{1}(z)$, let $P_{A}(z)=(1-A) P_{0}(z)+A P_{1}(z)$. If the zeros of $P_{0}$ and $P_{1}$ all lie in a circle $\mathscr{C}$ or on a line $L$, necessary and sufficient conditions are given for the zeros of $P_{A}(0 \leq A \leq 1)$ to all lie on $\mathscr{C}^{2}$ or $L$. This describes certain convex sets of monic $n$th degree polynomials having zeros in $\mathscr{C}$ or $L$. If the zeros of $P_{0}$ and $P_{1}$ lie in the unit disk and $P_{0}$ and $P_{1}$ have real coefficients, then the zeros of $P_{A}(0 \leq A \leq 1)$ lie in the disk $|z|<\cos (\pi / 2 n) /$ $\sin (\pi / 2 n)$. A set is described which includes the locus of zeros of $P_{A}(0 \leq A \leq 1)$ as $P_{0}$ and $P_{1}$ vary through all monic $n$th degree polynomials having all their zeros in a compact set $K$. When $K$ is path-connected, this locus is exactly the set described.

Given polynomials $P_{0}(z)$ and $P_{1}(z)$, let $P_{A}(z)$ denote the polynomial:

$$
P_{A}(z)=(1-A) P_{0}(z)+A P_{1}(z) .
$$

$P_{A}$ is defined for any complex value of $A$ and the zeros of $P_{A}(z)$ are continuous functions of $A$. In particular, if $A$ is varied through the reals between 0 and 1 , the locus of zeros of $P_{A}(z)$ is a network of paths in the plane starting at the zeros of $P_{0}(z)$ and terminating in the zeros of $P_{1}(z)$. If the degree of $P_{0}$ is higher than that of $P_{1}$ then some of the paths of zeros must tend to infinity as $A$ tends to one. It is the aim of this note to describe these loci of zeros when $P_{0}$ and $P_{1}$ are monic, have the same degree and are constrained to have their zeros on a circle, on a line or in a disk.

First, let $P_{0}$ and $P_{1}$ be real and have their zeros in $S^{1}=\{z \in$ $C:|z|=1\}$ where $C$ denotes the complex numbers. The following lemma gives a necessary and sufficient condition for the locus of zeros of $P_{A}(z)$. $(0 \leqq A \leqq 1)$ to be contained in $S^{1}$.

LEMma 1. Let $P_{0}(z)$ and $P_{1}(z)$ be real monic polynomials of degree $n$ with their zeros contained in $S^{1}-\{-1,1\}$. Denote the zeros of $P_{0}(z)$ by $w_{1}, w_{2}, \cdots, w_{n}$ and of $P_{1}(z)$ by $z_{1}, z_{2}, \cdots, z_{n}$ and assume:

$$
w_{i} \neq z_{j} \quad(1 \leqq i, j \leqq n)
$$

and

$$
\begin{aligned}
& 0<\arg \left(w_{i}\right) \leqq \arg \left(w_{j}\right)<2 \pi \\
& 0<\arg \left(z_{i}\right) \leqq \arg \left(z_{j}\right)<2 \pi \quad(1 \leqq i<j \leqq n)
\end{aligned}
$$


Let $\alpha_{i}$ be the smaller open arc of $S^{1}$ bounded by $w_{i}$ and $z_{i}(i=1, \cdots, n)$. Then the locus of zeros of $P_{A}(z)(0 \leqq A \leqq 1)$ is contained in $S^{1}$ if and only if the arcs $\alpha_{i}$ are disjoint.

Proof. If $P_{0}$ and $P_{1}$ are fixed, then for each $z \in C$ such that $P_{0}(z) \neq P_{1}(z)$ there is a unique value of $A=A(z)$ such that $P_{A(z)}(z)=0$. The function $A(z)$ is given by:

$$
A(z)=\frac{P_{0}(z)}{P_{0}(z)-P_{1}(z)}=\frac{1}{1-\frac{P_{1}(z)}{P_{0}(z)}}=\frac{1}{1-\frac{\left(z-z_{1}\right) \cdots\left(z-z_{n}\right)}{\left(z-w_{1}\right) \cdots\left(z-w_{n}\right)}}
$$

if $P_{0}(z) \neq 0$.

First assume that $P_{A}(z)$ has all its zeros in $S^{1}$ for $0 \leqq A \leqq 1$. When $A=0$, the zeros of $P_{A}(z)$ are the $w_{i}$. Perturbing $A$ from 0 to 1 will give a trajectory of zeros eminating from each $w_{i}$. Each trajectory will pass through a $z_{i}$ at $A=1$. Equation (*) implies that no $z$ can be a zero of $P_{A}(z)$ for two different values of $A$ (unless $P_{0}(z)=P_{1}(z)=0$ which is not the case here). Two trajectories can intersect only at $z$ 's which are multiple zeros of $P_{A}$ for some $A$. The set of all $z$ which are multiple zeros of $P_{A}(z)$ for some $A \in C$ is a finite set, as this is the set of $z \in C$ for which $P_{A}(z)$ and $P_{A}^{\prime}(z)$ are both zero. $P_{A}^{\prime}(z)=0$ implies $A(z)=P_{0}^{\prime}(z) /\left(P_{0}^{\prime}(z)-P_{1}^{\prime}(z)\right)$ if $P_{0}^{\prime}(z) \neq P_{1}^{\prime}(z)$ and equating this formula for $A(z)$ with that in $\left(^{*}\right)$ gives a polynomial that $z$ must satisfy if it is a multiple root of $P_{A}(z)$ for some $A$. Hence, two trajectories can cross but not coincide over a curve. If the trajectories are constrained to a circle, they can only intersect at their endpoints. The $n$ disjoint open arcs covered by these trajectories minus their endpoints are clearly the $\operatorname{arcs} \alpha_{i}$.

Now assume that the $\operatorname{arcs} \alpha_{i}$ are disjoint. Let $\theta_{i}$ denote the angle of the arc $\alpha_{i}$. Consider the quotients

$$
q_{i}=\frac{z-z_{i}}{z-w_{i}}
$$

and

$$
q_{n+1-i}=\frac{z-z_{n+1-i}}{z-w_{n+1-1}}=\frac{z-\bar{z}_{i}}{z-\bar{w}_{i}} .
$$

If $z \in S^{1}$ and $z \notin \alpha_{i} \cup \alpha_{n+1-i}$ then:

$$
\arg \left(q_{i}\right)= \pm \frac{\theta_{i}}{2}
$$

while 


$$
\arg \left(q_{n+1-i}\right)=\mp \frac{\theta_{i}}{2}
$$

Hence $\arg \left(q_{i} q_{n+1-i}\right)=0$ and $q_{i} q_{n+1-i}$ is a positive real. If $z \in S^{1}-$ $\mathrm{U}_{i=1}^{n} \bar{\alpha}_{i}$ then $P_{1}(z) P_{0}(z)$ is positive and except at the finite number of $z \in S^{1}$ where $P_{0}(z)=P_{1}(z), A(z)$ is real with either $A(z)<0$ or $A(z)>1$. If $z \in \alpha_{i}$ for some $i$, then for $j \neq i, q_{j} q_{n+1-j}$ is a positive real. On the other hand,

$$
\arg \left(q_{i}\right)= \pm\left(\pi-\frac{\theta_{i}}{2}\right)
$$

while

$$
\arg \left(q_{n+1-i}\right)= \pm \frac{\theta_{i}}{2}
$$

In this case $\arg \left(q_{i} q_{n+1-i}\right)=\pi$ so $P_{1}(z) / P_{0}(z)$ is negative and $A(z)$ is real with $0<A(z)<1$.

$A(z)$ is a continuous real-valued function of $z$ on each arc $\alpha_{i}$. $A(z)$ takes on the values 0 and 1 at the endpoints $w_{i}$ and $z_{i}$ of $\alpha_{i}$. $A(z)$ must then take on all values between 0 and 1 on each $\operatorname{arc} \alpha_{i}$. That is, for each $A(0 \leqq A \leqq 1)$ there is a zero of $P_{A}(z)$ in each arc $\alpha_{i}$. This accounts for all $n$ zeros of $P_{A}(z)$ so there can be no zeros of $P_{A}(z)$ outside $S^{1}$.

Note that a similar lemma holds for polynomials $P_{0}$ and $P_{1}$ having their zeros in any circle whose center is on the real line.

THEOREM 1. Let $\mathscr{C}$ be any circle whose center is on the real line and let $\gamma_{i}$ be open arcs in $\mathscr{C} \cap\{z \mid \operatorname{Im} z>0\}$ for $i=1, \cdots, k$. The set of (real) monic polynomials of degree $2 k$ with zeros $z_{1}, \bar{z}_{1}, \cdots$, $z_{k}, \bar{z}_{k}$ where $z_{i} \in \gamma_{i}(i=1, \cdots, k)$ is a convex set of polynomials if and only if the arcs $\gamma_{i}$ are disjoint.

Proof. All that remains is to consider what happens when $P_{0}$ and $P_{1}$ have zeros in common. In this case,

$$
\begin{aligned}
& P_{0}(z)=Q(z) \widetilde{P}_{0}(z), \\
& P_{1}(z)=Q(z) \widetilde{P}_{1}(z)
\end{aligned}
$$

and

$$
P_{A}(z)=Q(z)\left((1-A) \widetilde{P}_{0}(z)+A \widetilde{P}_{1}(z)\right)
$$

where $\widetilde{P}_{0}(z)$ and $\widetilde{P}_{1}(z)$ satisfy the conditions of Lemma 1. This lemma applied to $(1-A) \widetilde{P}_{0}(z)+A \widetilde{P}_{1}(z)$ implies the theorem.

Corollary 1. Let $P_{0}, P_{1}$ and $\alpha_{\imath}$ be as in Lemma 1. For each 
$z \in S^{1}$. Let $n(z)=\operatorname{card}\left\{\alpha_{i} \mid z \in \alpha_{i}\right\}$. For all $z \in S^{1}$ such that $P_{0}(z) \neq$ $P_{1}(z), z$ is a zero of $P_{A}(z)$ for some real value of $A=A(z)$ and $0 \leqq A(z) \leqq 1$ if and only if $n(z)$ is odd or $z$ is a zero of $P_{0}$ or $P_{1}$.

\section{Proof. This follows easily from the proof of Lemma 1.}

The techniques used in the proof of Lemma 1 applied to polynomials whose zeros lie on a straight line give the following result.

Theorem 2. Let $I_{j}(j=1, \cdots, n)$ be open intervals in a line $L \subseteq C$. The set of monic polynomials of degree $n$ having zeros $\zeta_{j}(j=1, \cdots, n)$ where $\zeta_{j} \in I_{j}$ is a convex set of polynomials if and only if the intervals $I_{j}$ are disjoint.

Proof. Let $P_{0}(z)$ and $P_{1}(z)$ have zeros $w_{1}, w_{2}, \cdots, w_{n}$ and $z_{1}, \cdots, z_{n}$, respectively, where $w_{j}$ and $z_{j}$ are in $L(j=1, \cdots, n)$. Assume that $L$ is directed and that the zeros $w_{i}$ and $z_{j}$ are ordered in this direction. Define intervals $\alpha_{j}$ and quotients $q_{j}$ as in Lemma 1 and its proof. If $P_{0}$ and $P_{1}$ are monic and $w_{i} \neq z_{j}(i, j=1, \cdots, n)$ then

$$
\begin{array}{llll}
\arg \left(q_{i}\right)=0 & \text { or } & 2 \pi & z \in L-\alpha_{i} \\
\arg \left(q_{i}\right)=\pi & \text { or } & -\pi & z \in \alpha_{i} .
\end{array}
$$

The arguments of Lemma 1 imply that the zeros of $P_{A}(0 \leqq A \leqq 1)$ are contained in $L$ if and only if the intervals $\alpha_{i}$ are disjoint and the theorem follows.

Theorem 2 is similar to a result of N. Obreschkoff [2] which states: Let $P(x)$ and $Q(x)$ be two polynomials without common zeros whose degrees differ by at most one. A necessary and sufficient condition that $P$ and $Q$ have only real zeros which separate each other is that the equations $a P+b Q=0$ have real zeros for all real $a$ and $b$. In the proof of Theorem 2, the zeros of $P_{0}$ and $P_{1}$ need not separate each other for $P_{A}(0 \leqq A \leqq 1)$ to have all its zeros on the line $L$. The zeros do, however, need to be "paired" which is the condition that the invervals $\alpha_{i}$ are disjoint. Theorem 2 can be restated in the flavor of Obreschkoff as follows.

THeOREM 2'. Let $P_{0}$ and $P_{1}$ be monic polynomials of the same degree. A necessary and sufficient condition that $P_{0}$ and $P_{1}$ have only paired zeros lying on one line $L$ is that the polynomials $P_{A}=$ $(1-A) P_{0}+A P_{1}$ have all their zeros on the line $L$ for $A$ real, $0 \leqq A \leqq 1$.

Lemma 1 is essentially Theorem 1 stated in this form. Theorem 
3 (below) can also be so formulated.

In Theorem 2, the polynomials $P_{0}$ and $P_{1}$ are not required to be real and there was no need of the symmetry obtained by having complex conjugate zeros. As linear transformations take circles into lines, there should be a version of Theorem 1 that does not require $P_{0}$ and $P_{1}$ to be real.

Theorem 3. Let 6 be a circle in the complex plane and let $\gamma_{i}(i=1, \cdots, n)$ be disjoint open arcs in $\mathscr{C}$. Let $z_{0} \in \mathscr{C}-\bigcup_{i=1}^{n} \gamma_{i}$. Then for any $w_{0} \in C, w_{0} \neq 0$, the set of polynomials $P$ having zeros $z_{i}(i=1, \cdots, n)$ where $z_{i} \in \gamma_{i}$ and satisfying $P\left(z_{0}\right)=w_{0}$ is a convex set of polynomials.

Proof. A transformation of the form

$$
w(z)=\alpha\left(\frac{z-\beta}{z-\delta}\right)
$$

will take a given circle through $\beta$ and $\delta$ onto a line, sending $\beta$ to the origin and $\delta$ to the point at infinity. The inverse of this transformation is given by

$$
z(w)=\delta\left(\frac{w-\alpha \beta / \delta}{w-\alpha}\right) .
$$

If $P(z)$ is the polynomial, $P(z)=a_{n} z^{n}+a_{n-1} z^{n-1}+\cdots+a_{1} z+a_{0}$ then

$$
\begin{aligned}
P(z(w))= & a_{n}\left(\delta\left(\frac{w-\alpha \beta / \delta}{w-\alpha}\right)\right)^{n}+\cdots+a_{1}\left(\delta\left(\frac{w-\alpha \beta / \delta}{w-\alpha}\right)\right)+a_{0} \\
= & \frac{1}{(w-\alpha)^{n}}\left[a_{n} \delta^{n}(w-\alpha \beta / \delta)^{n}+\cdots+a_{1} \delta(w-\alpha \beta / \delta)(w-\alpha)^{n-1}\right. \\
& \left.+a_{0}(w-\alpha)^{n}\right]=\frac{1}{(w-\alpha)^{n}} Q(w) .
\end{aligned}
$$

$Q(w)$ is a polynomial with leading coefficient $P(\delta)$ and $P(z)=0$ if and only if $Q(w(z))=0$. Take $\delta=z_{0}$ and $\mathscr{C}$ to be the given circle.

If $P_{0}$ and $P_{1}$ are polynomials in the set described in the statement of this theorem, let $Q_{0}, Q_{1}$ and $Q_{A}$ be the polynomials associated with $P_{0}, P_{1}$ and $P_{A}$ by the above. $Q_{A}=(1-A) Q_{0}+A Q_{1}$ and the proof of Theorem 2 applies to $Q_{A}$ as $Q_{0}$ and $Q_{1}$ have the same leading coefficient. $P_{A}(0 \leqq A \leqq 1)$ has zeros in the $\operatorname{arcs} \gamma_{i}$ and $P_{A}\left(\dot{z}_{0}\right)=w_{0}$ so $P_{A}$ is in the set described.

REMARK. Theorem 3 is not stronger than Theorem 1. Take, for example, $P_{0}(z)=z^{2}+z+1$ and $P_{1}(z)=z^{2}-z+1$ then $P_{0}(z) \neq P_{1}(z)$ for any $z \in S^{1}$. 
The results presented so far show, in particular, that if $P_{0}$ and $P_{1}$ are monic real polynomials of the same degree having zeros in $S^{1}$ or $R^{1}$ then any convex combination $P_{A}$ of $P_{0}$ and $P_{1}$ will have all its zeros in $S^{1}$ or $R^{1}$ if and only if the zeros of $P_{0}$ and $P_{1}$ are paired. There remains the question of where the zeros of $P_{A}$ must lie in general. A special case of a theorem of J.L. Walsh ([1] p. 77) says that if $P_{0}$ and $P_{1}$ are monic polynomials of degree $n$ with all their zeros contained in the disk $\{z \in C:|z| \leqq 1\}$, then all the zeros of $P_{A}(0 \leqq A \leqq 1)$ are contained in $\{z \in C:|z| \leqq 1 / \sin (\pi / 2 n)\}$.

This bound on the moduli of the zeros of $P_{A}(0 \leqq A \leqq 1)$ is optimal. If $|z|=1 / \sin (\pi / 2 n)$, construct the lines through $z$ which are tangent to the circle $S^{1}$ and let $w_{1}$ and $z_{1}$ be the points of tangency. Then $z$ will be a zero of $P_{1 / 2}$ if $P_{0}=\left(z-z_{1}\right)^{n}$ and $P_{1}=\left(z-w_{1}\right)^{n}$. If, however, $P_{0}$ and $P_{1}$ are real polynomials there is a slightly smaller bound on the moduli of zeros of $P_{A}(0 \leqq A \leqq 1)$.

THEOREM 4. Let $P_{0}$ and $P_{1}$ be real monic polynomials with their zeros contained in the unit disk $\{z \in C:|z| \leqq 1\}$. Then the zeros of $P_{A}(0 \leqq A \leqq 1)$ are contained in the disk

$$
\left\{z \in C:|z| \leqq \frac{\cos (\pi / 2 n)}{\sin (\pi / 2 n)}\right\}
$$

Proof. Let the zeros of $P_{0}$ and $P_{1}$ be denoted by $z_{1}, z_{2}, \cdots, z_{n}$ and $w_{1}, w_{2}, \cdots, w_{n}$ and assume that if $z_{i}\left(w_{i}\right)$ is not real then $z_{n+1-i}=$ $\bar{z}_{i}\left(w_{n+1-i}=\bar{w}_{i}\right)$. Let $q_{i}=\left(z-z_{i}\right) /\left(w-w_{i}\right)$. As in the proof of Lemma $1, z$ is a solution of $P_{A}$ for $0 \leqq A \leqq 1$, if and only if

$$
\arg \left(q_{1} q_{2} \cdots q_{n}\right)=\pi+2 k \pi \text { for some } k \in Z \text {. }
$$

The following two lemmas show that $\left|\arg \left(q_{i} q_{n+1-i}\right)\right|$ is maximal for $|z|$ fixed and greater than 1 when $z$ is pure imaginary and $\left\{z_{i}, w_{i}\right\}=$ $\{-1,+1\}=\left\{\bar{z}_{i}, \bar{w}_{i}\right\}$. In this case

$$
\left|\arg \left(q_{i}\right)\right|=2 \arctan \frac{1}{|z|},
$$

if $|z|>\cot (\pi / 2 n)$ then $1 /|z|<\tan (\pi / 2 n)$ and

$$
0<\arg \left(q_{1} \cdots q_{n}\right) \leqq 2 n \arctan \frac{1}{|z|}<2 n \arctan \left(\tan \frac{\pi}{2 n}\right)=\pi
$$

which is a contradiction.

Lemma 2. Let $a$ and $b$ be two points on a circle of radius 1 with center $c$. Let $p$ be at distance $r>1$ from $c$, then angle apb is maximal (minimal if negative) when angle acp equals angle pcb. 
Lemma 3. Let $a$ and $b$ be two points on a circle or radius 1 with center $c$ and let $p$ and $p^{\prime}$ be the two points on the perpendicular bisector of the segment ab at equal distances from $c$. Then angle $a p b+$ angle $b p^{\prime} a$ is maximal (minimal if negative) when $a b$ is a diameter of the circle.

To prove these two lemmas, I had to resort to the Law of Cosines and taking derivatives. The calculations are straightforward but tedious so I omit them here.

The following result shows what happens when the circle is replaced by a given compact set. The result is essentially the same as a theorem due to Nagy and generalized by Marden ([1] p. 32), though they state their results only for polynomials having their zeros in a given convex set.

Let $K \cong C$ be compact. Given $z \in C$, there is a minimal closed sector with vertex $z$ that includes $K$. Let $\theta(K, z)$ denote the angle of this sector. $0 \leqq \theta(K, z) \leqq 2 \pi(z \in C)$.

Theorem 5. Let $K \subseteq C$ be compact. The locus of zeros of $P_{A}$ $(0 \leqq A \leqq 1)$ as $P_{0}$ and $P_{1}$ run through all $n$th degree monic polynomials having all their zeros in $K$ is included in the set

$$
S(K, \pi / n)=\{z \in C \mid \theta(K, z) \geqq \pi / n\} .
$$

If $K$ is path-connected, this locus is exactly $S(K, \pi / n)$.

Proof. Let $q_{i}(i=1, \cdots, n)$ be defined as in the proof of Lemma 1. If $0<\theta(K, z)<\pi / n$ then $0 \leqq \arg \left(q_{i} \cdots q_{i}\right)<\pi$ and $z$ cannot be a zero of $P_{A}(0 \leqq A \leqq 1)$.

If $K$ is path-connected and $\theta(K, z) \geqq \pi / n$ there exist $z_{1}$ and $w_{1}$ in $S$ such that $\arg \left(\left(z-z_{1}\right) /\left(z-w_{1}\right)\right)=\pi / n$. $z$ is a zero of a $P_{A}(0 \leqq A \leqq 1)$ when $P_{0}(z)=\left(z-z_{1}\right)^{n}$ and $P_{1}(z)=\left(z-w_{1}\right)^{n}$.

Finally, we return to polynomials having their zeros in a line. The following result is stated for the interval $[-1,+1]$ in $R$ but it generalizes easily to polynomials having their zeros in any line segment in $C$.

Corollary 2. If $P_{0}$ and $P_{1}$ are monic $n$th degree polynomials having all their zeros in $[-1,+1]$ then the locus of zeros of $P_{A}(0 \leqq A \leqq 1)$ is included in the union of the two disks with diameter $\cot (\pi / 2 n)+\tan (\pi / 2 n)$ whose boundaries pass through the points -1 and +1 .

Proof. Observing that an inscribed angle on a circle is measured by half the arc it subtends shows that $S([-1,+1], \pi / n)$ is the 
union of the two disks described.

\section{REFERENCES}

1. Morris Marden, Geometry of Polynomials, Mathematical Surveys, Number 3, A.M.S. 1966.

2. N. Obreschkoff, Verteilung und Berechnung der Nullstellen Reeller Polynome, Deutscher Verlag der Wissenschaften, Berlin, 1963.

Received March 21, 1979 and in revised form August 28, 1979.

Northeastern UnIVERSiTY

Boston, MA 02115 


\section{PACIFIC JOURNAL OF MATHEMATICS}

\section{EDITORS}

DONALD BABBITT (Managing Editor)

University of Galifornia

Los Angeles, California 90024

Hugo RossI

University of Utah

Salt Lake City, UT 84112

C. C. MoOre AND ANDrew OGG

University of California

Berkeley, CA 94720

\section{J. DugundjI}

Department of Mathematics University of Southern California

Los Angeles, California 90007

R. Finn and J. Milgram

Stanford University

Stanford, California 94305

ASSOCIATE EDITORS

E. F. Beckenbachi

B. H. NeumanN

F. WOLF

K. YOSHIDA

\section{SUPPORTING INSTITUTIONS}

UNIVERSITY OF BRITISH COLUMBIA

CALIFORNIA INSTITUTE OF TECHNOLOGY

UNIVERSITY OF CALIFORNIA

MONTANA STATE UNIVERSITY

UNIVERSITY OF NEVADA, RENO

NEW MEXICO STATE UNIVERSITY

OREGON STATE UNIVERSITY

UNIVERSITY OF OREGON
UNIVERSITY OF SOUTHERN CALIFONIA

STANFORD UNIVERSITY

UNIVERSITY OF HAWAII

UNIVERSITY OF TOKYO

UNIVERSITY OF UTAH

WASHINGTON STATE UNIVERSITY

UNIVERSITY OF WASHINGTON

Printed in Japan by International Academic Printing Co., Ltd., Tokyo, Japan 


\section{Pacific Journal of Mathematics \\ Vol. 89 , No. 1 \\ May, 1980}

David Bressoud, A note on gap-frequency partitions ................ 1

John David Brillhart, A double inversion formula ................. 7

Frank Richard Deutsch, Günther Nürnberger and Ivan Singer, Weak

Chebyshev subspaces and alternation .......................

Edward Richard Fadell, The relationship between Ljusternik-Schnirelman

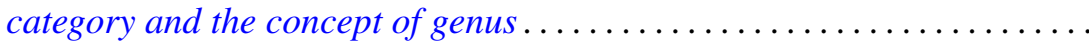

Harriet Jane Fell, On the zeros of convex combinations of polynomials.......

John Albert Fridy, An addendum to: "Tauberian theorems via block

dominated matrices" ..................................

Andrzej Granas, Ronald Bernard Guenther and John Walter Lee, Applications of topological transversality to differential equations. I. Some nonlinear

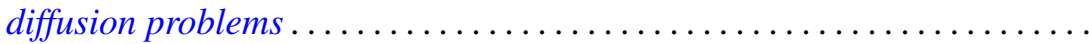

David E. Handelman and G. Renault, Actions of finite groups on self-injective

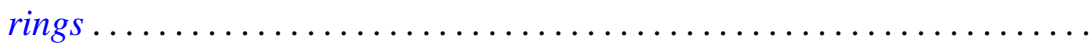

Michael Frank Hutchinson, Local $\Lambda$ sets for profinite groups .............

Arnold Samuel Kas, On the handlebody decomposition associated to a

Lefschetz fibration...

Hans Keller, On the lattice of all closed subspaces of a Hermitian space.....

P. S. Kenderov, Dense strong continuity of pointwise continuous

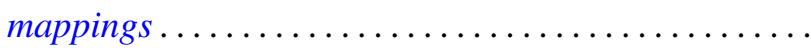

Robert Edward Kennedy, Krull rings.................

Jean Ann Larson, Richard Joseph Laver and George Frank McNulty,

Square-free and cube-free colorings of the ordinals ...

Viktor Losert and Harald Rindler, Cyclic vectors for $L^{p}(G)$

John Rowlay Martin and Edward D. Tymchatyn, Fixed point sets of

1-dimensional Peano continua...

Augusto Nobile, On equisingular families of isolated singularities ...

Kenneth Joseph Prevot, Imbedding smooth involutions in trivial bundles ...

Thomas Munro Price, Spanning surfaces for projective planes in four space.

Dave Riffelmacher, Sweedler's two-cocycles and Hochschild cohomology....

Niels Schwartz, Archimedean lattice-ordered fields that are algebraic over

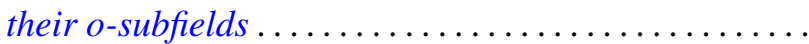

Chao-Liang Shen, A note on the automorphism groups of simple dimension groups.

Kenneth Barry Stolarsky, Mapping properties, growth, and uniqueness of

Vieta (infinite cosine) products ...

Warren James Wong, Maps on simple algebras preserving zero products. I.

The associative case ............................. 